\title{
Intrathecal Isoniazid for Refractory Tuberculous Meningitis with Cerebral Infarction
}

\author{
Yuko Nakatani ${ }^{1}$, Yutaka Suto ${ }^{1,2}$, Kazuki Fukuma ${ }^{1,2}$, Mika Yamawaki ${ }^{1}$, Ryoichi Sakata ${ }^{1,2}$, \\ Shotaro Takahashi ${ }^{1,2}$, Hiroyuki Nakayasu ${ }^{1}$ and Kenji Nakashima ${ }^{2}$
}

\begin{abstract}
A 30-year-old Vietnamese woman, about 19 weeks pregnant, was admitted for acute cerebral infarction with stenosis of the left middle cerebral artery (LMCA), tuberculous meningitis, and miliary tuberculosis. Treatment with heparin, quadruple anti-tuberculosis therapy, and dexamethasone afforded prompt symptomatic improvement. However, she delivered a stillbirth, after which there was recurrence of acute cerebral infarction with LMCA occlusion, sinus thrombosis, and cranial base inflammation. A thrice-weekly $100 \mathrm{mg}$ dose of intrathecal isoniazid (INH) improved the signs of meningeal inflammation. The patient was discharged ambulatory after 7 months. In refractory tuberculous meningitis, multimodal therapy with intrathecal INH and steroids should be considered.
\end{abstract}

Key words: intrathecal isoniazid, tuberculous meningitis, cerebral infarction, pregnancy

(Intern Med 56: 953-957, 2017)

(DOI: 10.2169/internalmedicine.56.6945)

\section{Introduction}

Tuberculous meningitis is a serious disease with high rates of mortality and residual neurologic deficits (1). Although the British Infection Society has recommended treatment regimens (2), additional high-strength modalities may be needed in some serious cases. We herein report a case of tuberculous meningitis in which remission was achieved not with the recommended treatment but with intrathecal administration of isoniazid (INH).

\section{Case Report}

The patient was a 30-year-old Vietnamese woman with no relevant medical history who was studying in Japan. She presented with common cold symptoms for 2 weeks and a fever of about $38^{\circ} \mathrm{C}$, followed by right hemiplegia. These prompted consultation and subsequent admission to our hospital. The patient had undergone fertility treatment at a private obstetrics-gynecology clinic and was about 19 weeks pregnant. She had hyperemesis with a poor nutritional status, however, no weight gain during pregnancy was observed.

The general physical findings were as follows: height 157 $\mathrm{cm}$, weight $52 \mathrm{~kg}$, body mass index 21.1, blood pressure $116 / 57 \mathrm{mmHg}$, heart rate $128 / \mathrm{min}$, respiration rate $30 / \mathrm{min}$, body temperature $38.0^{\circ} \mathrm{C}$, and oxygen saturation of $95 \%$. There were no pertinent thoracic or abdominal findings, except for the signs of pregnancy. The neurologic findings were lucid consciousness, spontaneous pain in the posterior cervical region, nuchal rigidity, right central facial palsy, right hemiplegia (Brunnstrom stage I in the arm and fingers, Brunnstrom stage II in the leg), hyperreflexia of the right upper and lower limbs, and right leg sensory deficit. Blood inflammatory markers showed a white blood cell count of $6,920 / \mu \mathrm{L}$ and C-reactive protein of $3.42 \mathrm{mg} / \mathrm{dL}$. Blood biochemistry results indicated mild liver dysfunction (aspartate aminotransferase $43 \mathrm{IU} / \mathrm{L}$, alanine aminotransferase $46 \mathrm{IU} / \mathrm{L}$ ) and undernutrition, with albumin at $2.3 \mathrm{~g} / \mathrm{dL}$. There were no abnormal findings related to the kidney or pancreas function. Testing for human immunodeficiency virus was negative.

The cerebrospinal fluid (CSF) findings were as follows:

${ }^{1}$ Department of Neurology, Tottori Prefectural Central Hospital, Japan and ${ }^{2}$ Department of Brain and Neurosciences, Division of Neurology, Faculty of Medicine, Tottori University, Japan

Received for publication December 25, 2015; Accepted for publication July 26, 2016

Correspondence to Dr. Yutaka Suto, sutoyutaka.i@gmail.com 

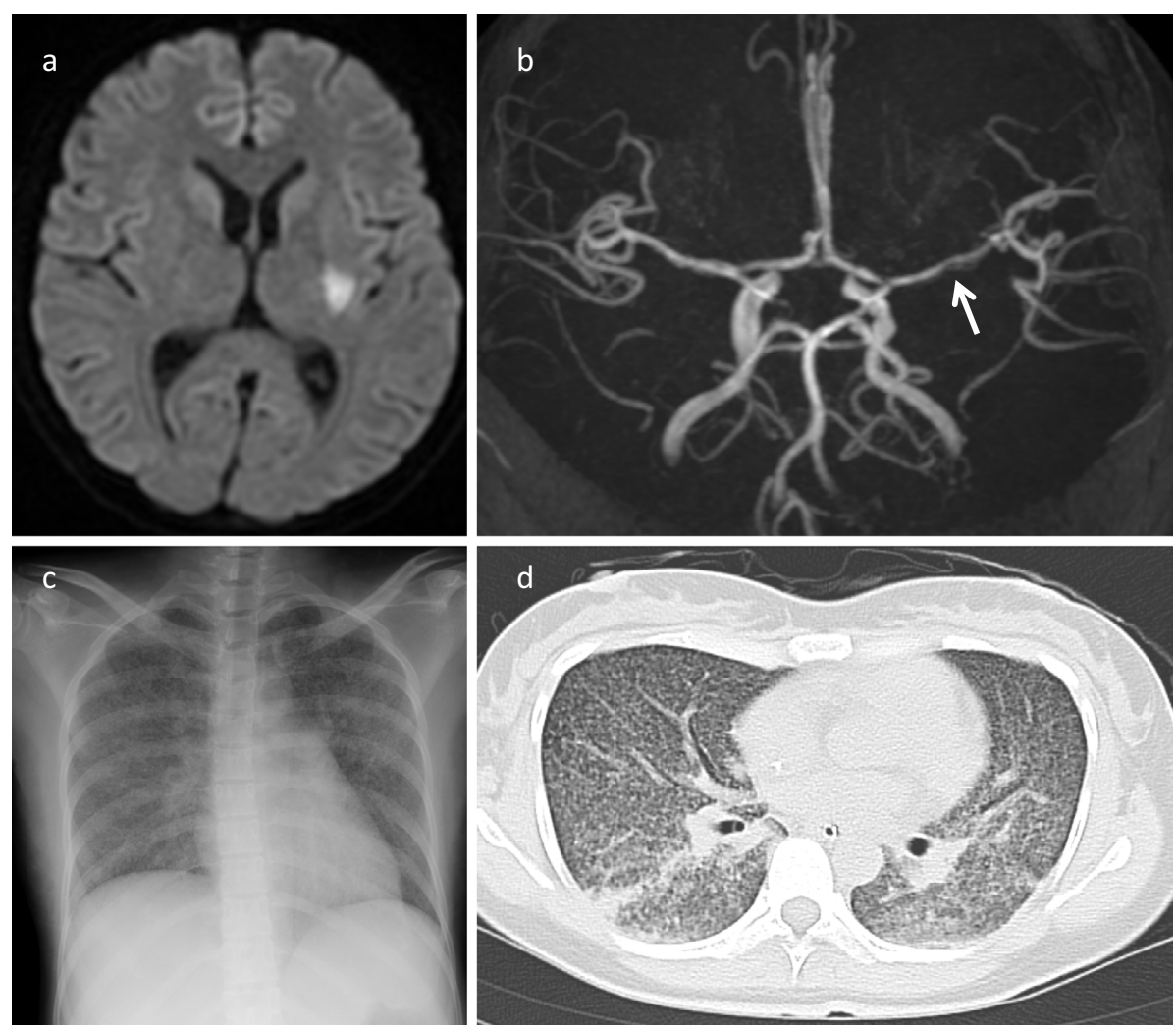

Figure 1. Radiologic imaging findings in a 30-year-old woman with refractory tuberculous meningitis. (a) Cranial MRI diffusion-weighted image shows acute cerebral infarction of the left putamen. (b) MRA shows stenosis in the horizontal segment of the left middle cerebral artery (arrow). (c) Chest radiograph shows miliary shadows in both lung fields. (d) On chest CT, there were diffuse ground glass opacities in both lung fields. MRI: magnetic resonance imaging, MRA: magnetic resonance angiography, CT: computed tomography

pale yellow color, initial pressure $290 \mathrm{mmH}_{2} \mathrm{O}$, final pressure $150 \mathrm{mmH}_{2} \mathrm{O}$, cell count $100 / \mu \mathrm{L}$ with a lymphocyte-topolymorphonuclear leukocyte ratio of $67: 33$, protein 229 $\mathrm{mg} / \mathrm{dL}$, sugar $11 \mathrm{mg} / \mathrm{dL}$, and positive Ziehl-Neelsen staining. Cranial magnetic resonance imaging (MRI) on diffusionweighted images revealed acute cerebral infraction of the left putamen; magnetic resonance angiography (MRA) revealed stenosis of the left middle cerebral artery (LMCA) (Fig. 1a and b). Ground glass opacities in both lung fields were noted on chest radiograph and computed tomography (CT) (Fig. 1c and d). Cerebral infarction secondary to vasculitis from tuberculous meningitis and miliary tuberculosis were suspected.

Fig. 2 shows the clinical course of the patient's hospitalization. Treatment was started with dexamethasone (19.6 mg/ day) and four anti-tuberculosis drugs: INH (200 mg/day), rifampicin (RFP) (450 mg/day), ethambutol (EB) $(750 \mathrm{mg} /$ day), and pyrazinamide (PZA) (1.2 g/day). Because bacterial meningitis could not be ruled out, meropenem ( $6 \mathrm{~g} /$ day) was also administered. Taking into consideration the patient's pregnancy, acute cerebral infarction was treated with continuous heparin infusion at 10,000 units/day. Based on positive tubercle bacilli DNA on polymerase chain reaction and positive culture for Mycobacterium tuberculosis, the patient was given a definitive diagnosis of tuberculous meningitis.
After five days of treatment, liver dysfunction ensued. When the liver dysfunction improved after a week, the administration of the four drugs was continued, with PZA being replaced by levofloxacin (LVFX) (500 mg/day). The fever and right hemiplegia promptly improved. Although ZiehlNeelsen staining was performed only once and showed positivity and susceptibility to all anti-tuberculous drugs, the presence of Mycobacterium tuberculosis in the CSF was sustained for up to one month, despite the administration of anti-tuberculosis drugs.

On hospital day 25, at 22 weeks and 1 day of gestation, the patient gave birth to a stillborn. Examination of the uterine contents revealed tubercule bacilli, confirming an intrauterine infection. After the stillbirth, the fever and meningitis signs recurred. Despite treatment with 5 anti-tuberculosis agents (with the addition of streptomycin at $1 \mathrm{~g} /$ day) and restarting dexamethasone administration (19.6 mg/day), no improvement in the signs of meningeal irritation was observed. On hospital day 33, aphasia and right hemiplegia appeared; on hospital day 37, right abducens nerve palsy developed. Repeat cranial MRI revealed recurrent cerebral infarction on the left parietal lobe; MRA showed peripheral occlusion of the horizontal segment of the LMCA. Findings indicative of inflammation of the cranial base and lateral sinus thrombosis were also observed (Fig. 3). These were treated with 


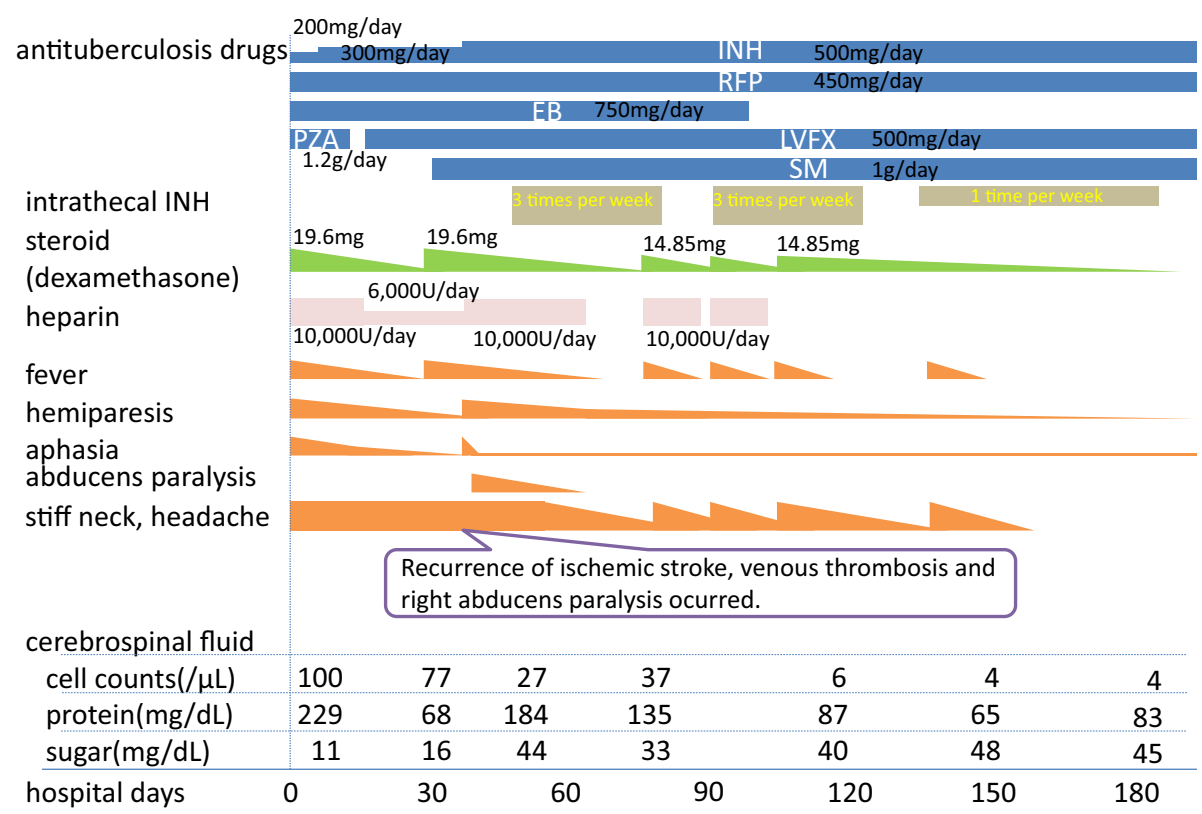

Figure 2. Clinical course of a 30-year-old woman with refractory tuberculous meningitis. INH: isoniazid, RFP: rifampicin, EB: ethambutol, PZA: pyrazinamide, LVFX: levofloxacin, SM: streptomycin
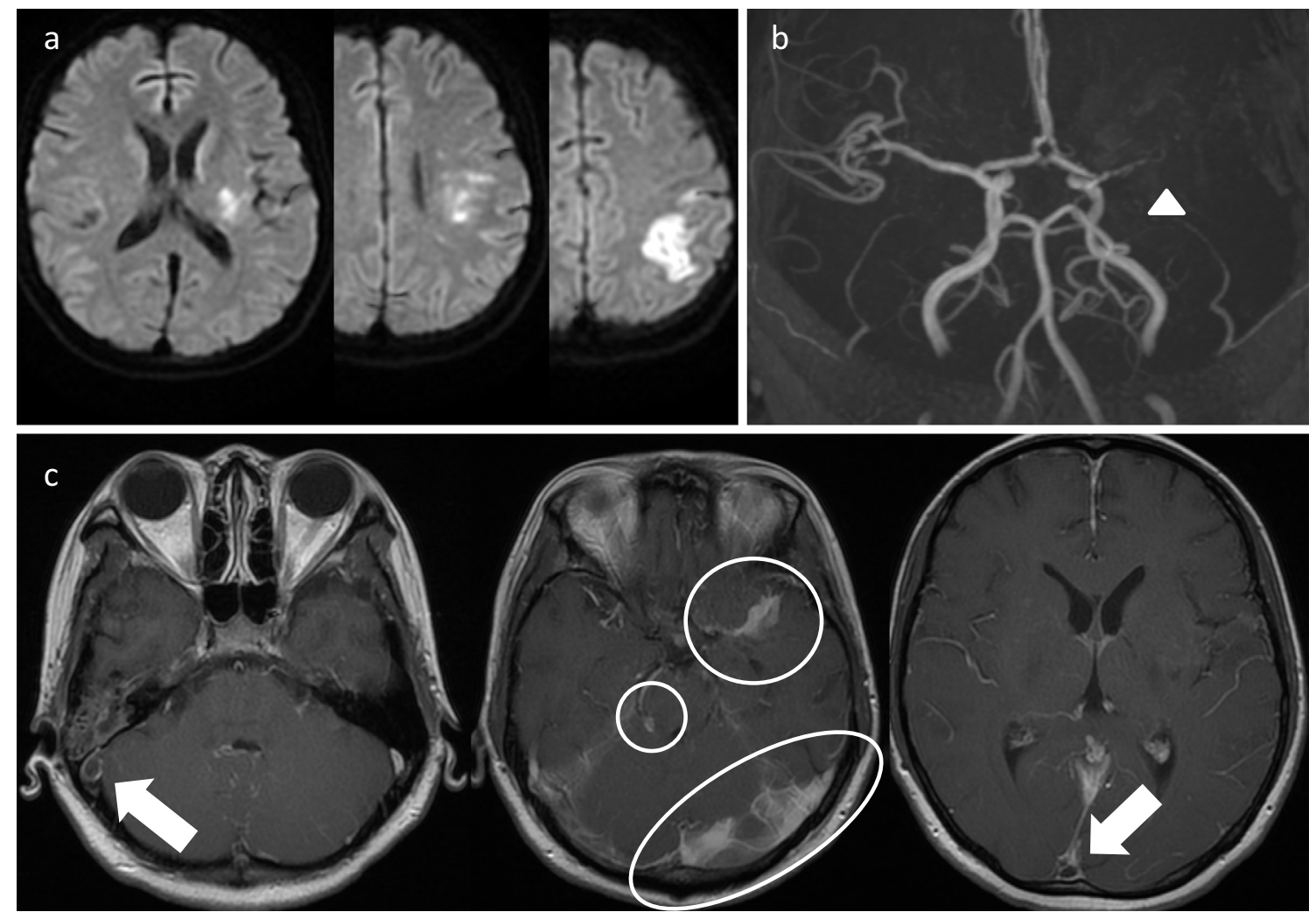

Figure 3. Cranial MRI findings of recurrent cerebral infarction in tuberculous meningitis. (a) Diffusion-weighted images show infarcted area in the left posterior limb of the internal capsule, left corona radiata, and left parietal lobe. (b) MRA shows occlusion of the left middle cerebral artery (arrow head). (c) Enhanced T1-weighted images show inflammatory changes in the base of the brain (encircled area) and venous sinus thrombosis (arrow). MRI: magnetic resonance imaging, MRA: magnetic resonance angiography 


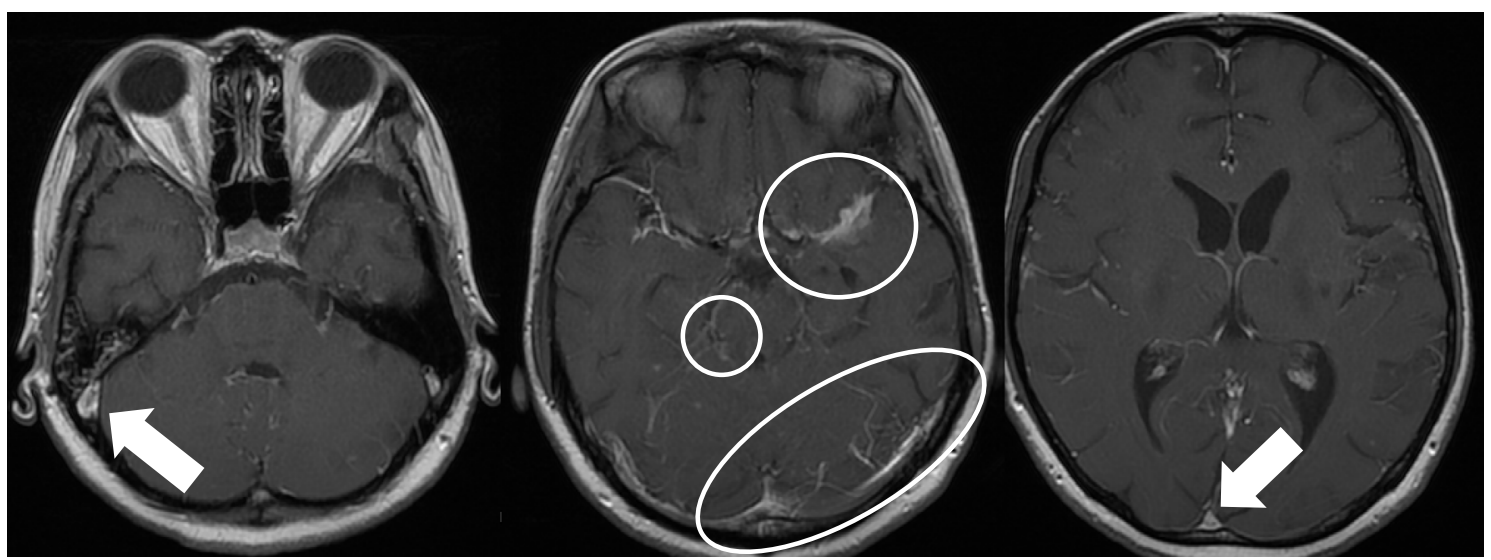

Figure 4. Cranial MRI after intrathecal administration of INH for tuberculous meningitis complicated by cerebral infarction. Enhanced T1-weighted images show improvement of the inflammatory changes in the base of the brain (encircled area) and resolution of sinus thrombosis (arrow). MRI: magnetic resonance imaging, INH: isoniazid

edaravone and heparin; administration of aspirin $(100 \mathrm{mg} /$ day) was also continued to prevent recurrence.

However, the meningitis symptoms persisted and did not improve despite increasing the INH dosage from $300 \mathrm{mg}$ / day to $500 \mathrm{mg} /$ day. Therefore, intrathecal administration of $100 \mathrm{mg}$ of INH 3 times per week was started. Furthermore, $15 \mathrm{~mL}$ of CSF was intermittently drained by lumbar puncture to relieve intracranial pressure. The signs of meningeal irritation started to improve after starting intrathecal INH. Serial cranial MRI indicated resolution of the venous sinus thrombosis and reduction of the inflammatory findings in the cranial base (Fig. 4). On hospital day 98, the visual acuity on the left decreased, suggesting left optic neuritis caused by inflammation of the cranial base. As the possibility of optic neuropathy could not be ruled out, EB was discontinued. Intrathecal INH administration was tapered to once a week and eventually was discontinued as the patient's symptoms improved.

The patient was discharged for home on hospital day 191. Three anti-tuberculosis drugs (INH, RFP, and LVFX) were continuously administered for 1 year. The residual neurologic deficits were mild aphasia, decreased visual acuity on the left side, and hypoesthesia in both legs, which was suspected to be secondary to INH. Nevertheless, the patient was able to independently engage in her activities of daily living. Cranial MRI 2 years after onset showed that the occlusion in the horizontal segment of the LMCA was unchanged; therefore, the administration of aspirin (100 mg/ day) was continued to prevent the recurrence of cerebral infarction. Approximately 2.5 years later, the patient returned to her home country. She was able to walk without assistance and carry out her usual duties and activities.

\section{Discussion}

Tuberculous meningitis causes inflammatory changes in the base of the brain, leading to vasculitis, cranial polyneuri- tis, and obstructive hydrocephalus (3). Cerebral infarction as a complication is widely known to be associated with a poor prognosis (4).

In the present case, we referred to the recommendations of the British Infection Society (2) and administered a fourdrug combination of INH, RFP, EB, and PZA. Although the guidelines for treating tuberculosis in pregnant patients state that PZA should be avoided as much as possible because its safety has not been established (5), we decided to continue PZA to save the mother's life, as this was a severe case of tuberculosis. Based on a report by Thwaites et al. (6), we also administered corticosteroids. The recommended treatment led to temporary improvement in the symptoms. However, this was a severe case of recurrent meningitis and cerebral infarction, sinus thrombosis, and cranial neuropathy, for which sustained improvement was only achieved by additional intrathecal administration of INH.

There have been only a small number of reports on the use of intrathecal INH. Marked improvement was noted in a case of refractory tuberculous meningitis after thrice-weekly intrathecal administration of $100 \mathrm{mg} /$ day of INH (7). This therapy was also proven to achieve remission in a patient with tuberculous meningitis and liver dysfunction, where the oral administration of high-dose INH was not possible (8). In other reports, two patients were able to return to society without any serious residual effects after combination of intrathecal INH and steroid pulse therapy (9). These findings suggest that intrathecal administration of INH may contribute to an improved prognosis in refractory tuberculous meningitis.

After administration, INH rapidly diffuses into the CSF and reaches peak concentrations in excess of $3 \mathrm{mg} / \mathrm{L}$, which is over 30 times its minimal inhibitory concentration (MIC) against Mycobacterium tuberculosis, within 4 hours $(10,11)$. INH is metabolized in the liver via the N-acetyltransferase 2 (NAT2), which is a polymorphic gene with enzymatic activity that can be altered by the presence of single nucleotide 
polymorphisms (SNPs) in its coding region (12). Three major genetically determined phenotypes are observed: rapid, intermediate, and slow acetylators $(12,13)$. Among Brazilian patients with tuberculosis, $72.8 \%$ were found to be fast acetylators (14). A meta-analysis showed a significant association between the slow acetylator genotype of NAT2 and antituberculosis drug-induced hepatotoxicity; significant results were also found in East Asians, South Asians, Brazilians, and Middle Eastern populations, but none in Caucasians (15). This implies that the efficacy of INH may be based on NAT2 gene polymorphism and race.

In our case, the resistance to standard tuberculous therapy and the effectiveness of intrathecal INH were probably due to 1) disturbance of CSF circulation, 2) decline in INH penetration to the focal lesion, and 3 ) the possibility that the patient was a rapid acetylator of NAT2, although we did not investigate the polymorphism status in the present case. Overall, these findings suggest that refractory tuberculous meningitis or tuberculous meningitis complicated by antituberculosis drug-induced hepatotoxicity may be indications for treatment with intrathecal INH.

This case exhibited recurrent meningitis symptoms and required administration of corticosteroids over several months. Corticosteroids can improve CSF findings, reduce intracranial pressure, and decrease inflammation $(16,17)$. It has also been suggested that corticosteroids be routinely used in HIV-negative people with tuberculous meningitis to reduce the risk of death and disabling residual neurologic deficit among survivors (1). Thwaites et al. (6) reported a protocol involving steroid discontinuation after 4 weeks. However, in refractory cases with persistent meningitis symptoms longer than 4 weeks, as exemplified by our patient, we believe that prolonged administration of corticosteroids can be considered.

In conclusion, multimodal therapy, including intrathecal administration of INH and systemic steroids, should be considered for cases of refractory tuberculous meningitis.

The authors state that they have no Conflict of Interest (COI).

\section{References}

1. Prasad K, Singh MB. Corticosteroids for managing tuberculous meningitis. Cochrane Database Syst Rev (1): CD002244, 2008.

2. Thwaites G, Fisher M, Hemingway C, Scott G, Solomon T, Innes J; British Infection Society. British Infection Society guidelines for the diagnosis and treatment of tuberculosis of the central nervous system in adults and children. J Infect 59: 167-187, 2009.

3. Dastur DK, Manghani DK, Udani PM. Pathology and pathogenetic mechanisms in neurotuberculosis. Radiol Clin North Am 33: 733, 1995.

4. Wasay M, Farooq S, Khowaja ZA, et al. Cerebral infarction and tuberculoma in central nervous system tuberculosis: frequency and prognostic implications. J Neurol Neurosurg Psychiatry 85: 12601264, 2014.

5. Hamadeh MA, Glassroth J. Tuberculosis and pregnancy. Chest 101: 1114-1120, 1992.

6. Thwaites GE, Nguyen DB, Nguyen HD, et al. Dexamethasone for the treatment of tuberculous meningitis in adolescents and adults. N Engl J Med 351: 1741-1751, 2004.

7. Takahashi T, Ogawa K, Sawada S, et al. A case of refractory tuberculous meningitis markedly improved by intrathecal administration of isoniazid (INH). Rinsho Shinkeigaku 43: 20-25, 2003 (in Japanese, Abstract in English).

8. Danielides IC, Constantoulakis M, Daikos GK. Hepatitis on high dose isoniazid: reintroduction of the drug in severe tuberculous meningitis. Am J Gastroenterol 148: 650-655, 1983.

9. Takahashi I, Yamada M, Matsushima M, et al. Treatment of intractable tuberculous meningitis using intrathecal isoniazid administration and steroid pulse therapy; a report of two cases. Rinsho Shinkeigaku 52: 551-556, 2012 (in Japanese, Abstract in English).

10. Thwaites GE, van Toorn R, Schoeman J. Tuberculous meningitis: more questions, still too few answers. Lancet Neurol 12: 9991010, 2013.

11. Ellard GA, Humphries MJ, Allen BW. Cerebrospinal fluid drug concentrations and the treatment of tuberculous meningitis. Am Rev Respir Dis 148: 650-655, 1993.

12. Teixeira RL, Morato RG, Cabello PH. Genetic polymorphisms of NAT2, CYP2E1 and GST enzymes and the occurrence of antituberculosis drug-induced hepatitis in Brazilian TB patients. Mem Inst Oswaldo Cruz 106: 716-724, 2011.

13. Kinzig-Schippers M, Tomalik-Scharte D, Jetter A, et al. Should we use $\mathrm{N}$-acetyltransferase type 2 genotyping to personalize isoniazid doses? Antimicrob Agents Chemother 49: 1733-1738, 2005.

14. Possuelo LG, Castelan JA, de Brito TC, et al. Association of slow $\mathrm{N}$-acetyltransferase 2 profile and anti-TB drug-induced hepatotoxicity in patients from Southern Brazil. Eur J Clin Pharmacol 64: 673-681, 2008.

15. Du H, Chen X, Fang Y, et al. Slow N-acetyltransferase 2 genotype contributes to anti-tuberculosis drug-induced hepatotoxicity: a meta-analysis. Mol Biol Rep 40: 3591-3596, 2013.

16. Feldman S, Behar AJ, Weber D. Experimental tuberculous meningitis in rabbits. AMA Arch Pathol 65: 343-354, 1958.

17. Parsons M. Tuberculous Meningitis: Tuberculomas and Spinal Tuberculosis: A Handbook for Clinicians. Oxford: Oxford University Press, 1988: 32-62.

The Internal Medicine is an Open Access article distributed under the Creative Commons Attribution-NonCommercial-NoDerivatives 4.0 International License. To view the details of this license, please visit (https://creativecommons.org/licenses/ by-nc-nd/4.0/).

(C) 2017 The Japanese Society of Internal Medicine http://www.naika.or.jp/imonline/index.html 